\title{
A Novel Low RCS Design Method for X-Band Vivaldi Antenna
}

\author{
XiaoXiang He, Teng Chen, and Xin Wang \\ College of Electronic and Information Engineering, Nanjing University of Aeronautics and Astronautics, \\ 29 Yudao Streat, Baixia District, Nanjing 210016, China
}

Correspondence should be addressed to Teng Chen, ctdziwo@sina.com

Received 17 February 2012; Accepted 6 June 2012

Academic Editor: Dalia N. Elshiekh

Copyright (C) 2012 XiaoXiang He et al. This is an open access article distributed under the Creative Commons Attribution License, which permits unrestricted use, distribution, and reproduction in any medium, provided the original work is properly cited.

\begin{abstract}
A novel low radar cross-section (RCS) design method is proposed, and its application on Vivaldi antenna that covers the entire X band is investigated. According to the difference of the current distribution on the radiator when the antenna radiates or scatters, the shape of the metal radiator is modified, so that maximally 19.2 dBsm RCS reduction is achieved which satisfied radiation performance. Simulated and measured results about gain, $S_{11}$, and RCS are presented. As a result, the effectiveness of the presented low RCS design method is validated.
\end{abstract}

\section{Introduction}

The nose cone of an aircraft is the most threating area for radar detection, but the electromagnetic scattering of antenna array belongs to the fire control system in this area is usually very strong, which result in the importance of stealth design of antenna. Vivaldi antennas [1-3] are widely used in fire control system due to broad bandwidth and small physical dimension. Therefore, the stealth design of Vivaldi antenna is highly desirable in many prospective airborne applications.

Many methods [4-11] have been proposed to reduce the radar cross-section (RCS) of microstrip antennas. Usually, RCS reduction will increase the complexity of the antenna system or degrade the radiation performance of the antenna. Typical performance-degradation effects include gain decreasing, resonant frequency shifting, and bandwidth narrowing. Li and Liu $[4,5]$ proposed a stealth method with maximally $27 \mathrm{dBsm}$ RCS reduction at a specific frequency with two short-circuit pins loaded on each microstrip unit and two H-shaped slots cut. However, the RCS reduction occurred only at a relatively narrow frequency band and angle range. Another effective method, investigated by Jang et al. $[6,7]$, uses the electromagnetic band gap (EBG) structure and achieves a $10 \mathrm{dBsm}$ RCS reduction out of the working band while keeping the RCS within the operating band almost unchanged. The same effect can also be acquired with frequency-selective surface (FSS) on radomes. Other methods [8-10] such as Fractal slots, defected ground structures (DGSs), or holly-leaf-shaped designs, also can reduce RCS in angle scope vertical to the antenna in the operating band, but all the methods are hard to be applied to Vivaldi antenna for its threating direction is almost parallel to the antenna.

Several RCS reduction schemes have also been developed for Vivaldi antennas. Zhang et al. [11] introduced a phaseswitched screen (PSS) boundary, which enables the dynamic control of RCS. Jiang fu et al. [12] investigated RCS a dual-index Vivaldi antenna which achieves a maximally of $25 \mathrm{dBsm}$ RCS reduction, but the dispersion of the measured gain is decreased.

In this paper, we propose a novel low RCS design method that specifically applies to the X-band Vivaldi antenna. The design is based on the analysis of the antenna current distribution in the radiating and scattering status, respectively. Then we modify the configuration of the metal patch to achieve maximally $19.2 \mathrm{dBsm}$ RCS reduction. Simulation and measurement results are provided to demonstrate the excellent radiation and stealth performance that are achieved with this design.

\section{Stealth Design Scheme}

A typical Vivaldi antenna [13-15] without any low RCS design is shown in Figure 1. The antenna is printed on the 


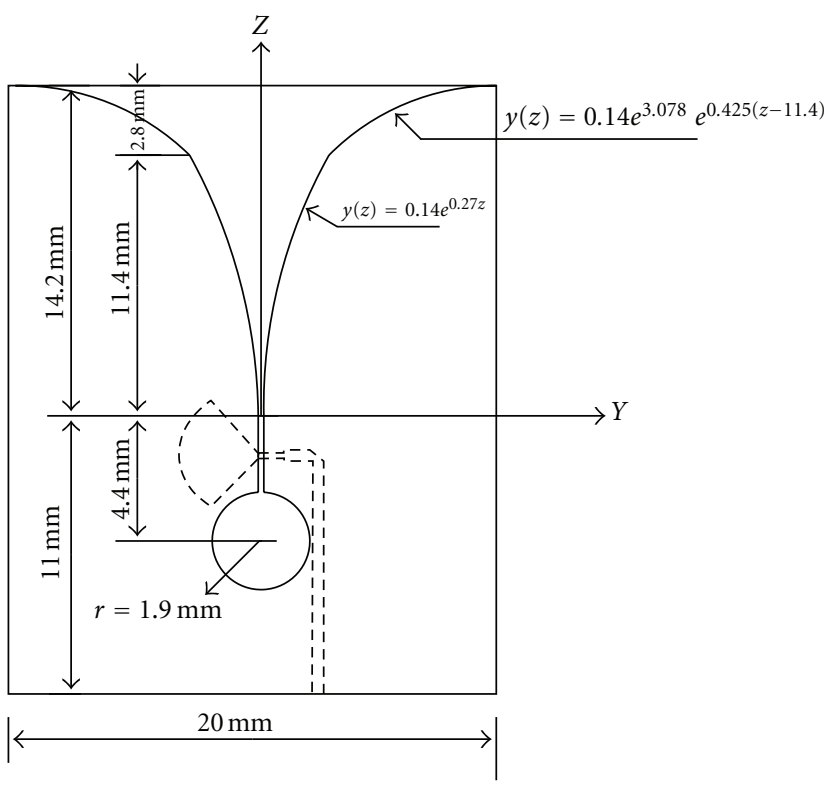

(a) The front view

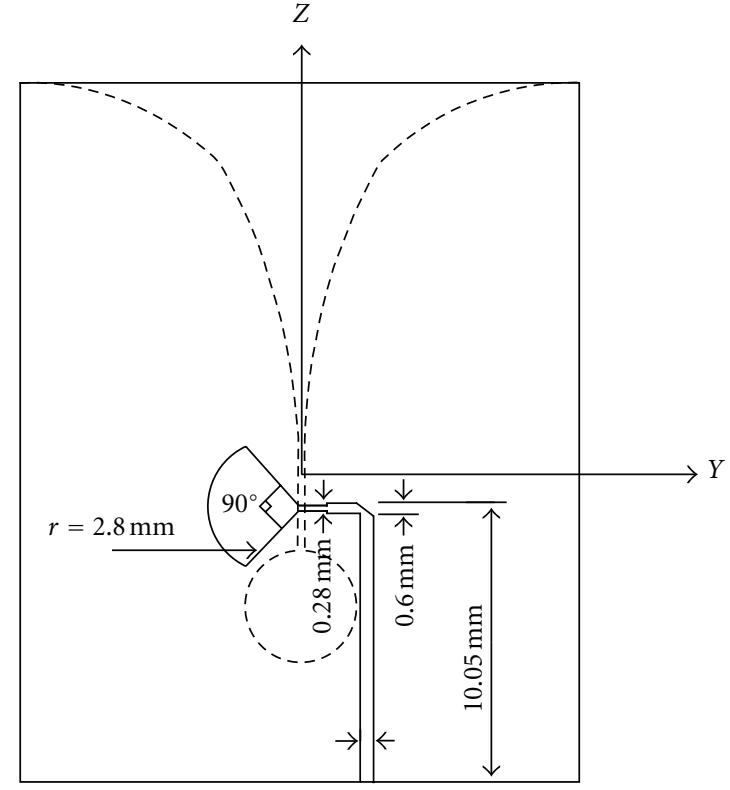

(b) The back view

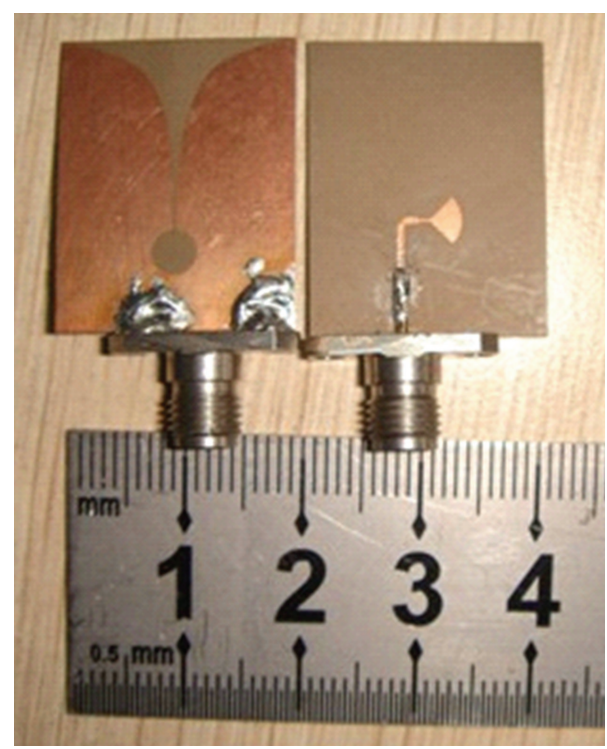

(c) Fabricated original antenna

Figure 1: Structure of original Vivaldi antenna.

two sides of a TF-2 $\left(\varepsilon_{r}=10.2\right)$ substrate with a thickness of $0.635 \mathrm{~mm}$. On one side of the substrate is the metal patch consisting of a tapered slot line and a transmission line as illustrated by the solid lines of in Figure 1(a). On the other side, there is a feeding structure as illustrated by the solid lines of in Figure 1(b). For better illustration, dotted lines are employed in both views to indicate the position of the structures on the opposite side. The parameters marked in the figure are optimized with HFSS to match the feeding port to $50 \Omega$. The photograph of the fabricated original antenna is shown in Figure 1(c). The measured and Generally speaking, the scattering of an antenna can be divided into two parts $[16,17]$. One part is the so-called structural mode scattering that excludes the effect of the antenna's receiving channel.
This part of scattering is almost the same as the scattering of a common scatterer. The other part is the mode scattering that is caused by the reradiation of the reflected power from the receiving channel due to the impedance mismatching. Nevertheless, the mode scattering of a well-matched antenna is usually much smaller than the structural mode scattering. Take the Vivaldi antenna in Figure 1 as an example, the total RCS is about $-33 \mathrm{dBsm}$ at $10 \mathrm{GHz}$ as simulated with HFSS while the mode scattering RCS is estimated to be about $-75 \mathrm{dBsm}[18]$ which is negligibly small. Therefore, in this paper, we mainly focus on the structural mode stealth design of the Vivaldi antenna.

The purpose of a stealth design is to decrease RCS of an antenna while maintaining the antenna's radiation 


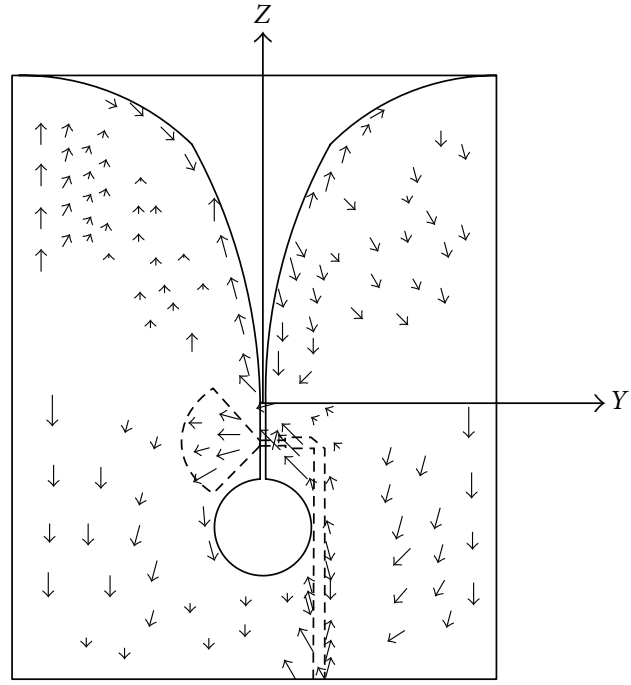

(a) Scattering mode

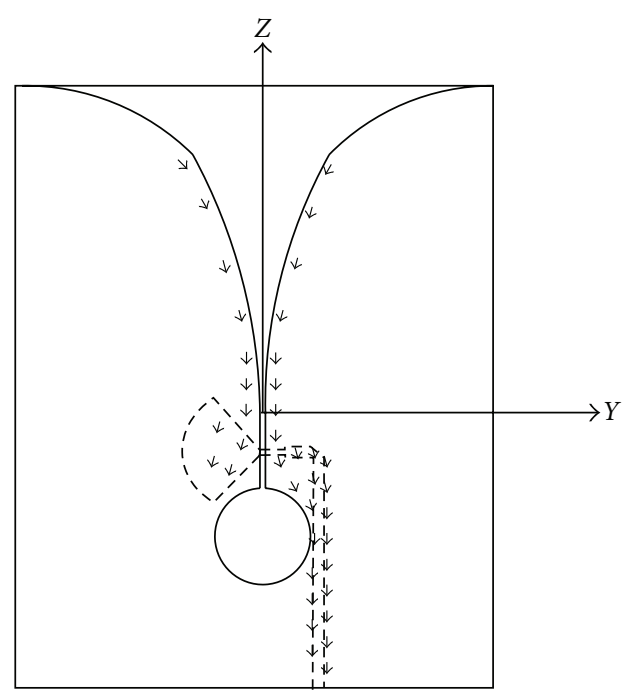

(b) Radiation mode

Figure 2: Current distribution of metal patch.

performance, which is usually conflict and should be balanced between scattering model and radiation model. In our stealth design method, the balance is well achieved by utilizing the difference between the current distributions of the antenna in the two modes. At $10 \mathrm{GHz}$, the induced current distribution due to scattering and radiation are shown in Figures 2(a) and 2(b), respectively. It can be seen from Figure 2(a) that the current distributes throughout the entire metal patch when the antenna is incident by a plane wave from $\theta^{i}=0^{\circ}$ and $\phi^{i}=0^{\circ}$. On the other hand, when the antenna is in the radiating mode, the current is mainly along the feeding structure and transmission line as is seen in Figure 2(b). Consequently, it can be found that there are large areas of the metal patch have no contribution to the radiation but play an important role in the scattering. The main idea of the stealth design is to, therefore, remove these metal areas in

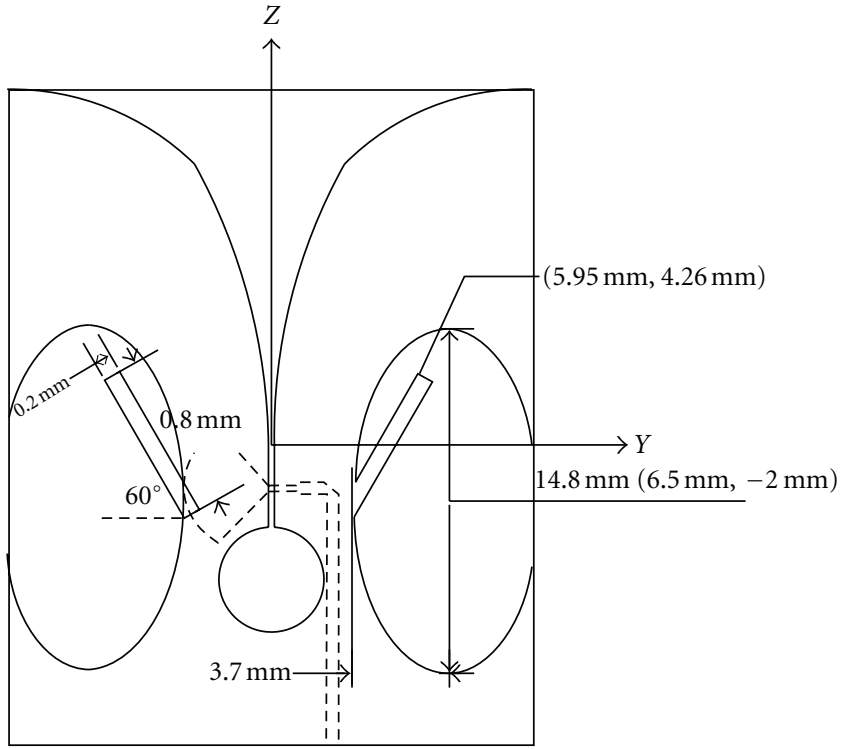

FIgURE 3: Structure of stealth design.

a way that decreases the RCS without sacrificing the radiation characteristics.

Based on the aforementioned idea, two ellipses are symmetrically cut out from the metal patch to ensure the maximum metal reduction to reduce RCS. However the large area cutdown on the metal patch will unavoidably result in slight current distribution changing in the radiation model, which will lead to high side lobe and dispersive gain in a wide frequency range. To prevent the lateral radiation, a rectangle strip is placed within the area of the two cut ellipses and connected to the remaining part of the metal patch. The rectangle strip's length is $7.8 \mathrm{~mm}$ and its resonant frequency is nearly $3 \mathrm{GHz}$, and then we can have two higher harmonics at $9 \mathrm{GHz}$ and $12 \mathrm{GHz}$. Through HFSS simulation and optimization, we arrive at a final design of the stealth antenna as shown in Figure 3. The stealth antenna is fabricated and shown in Figure 4.

\section{Result and Discussion}

To validate the stealth design method presented in the previous section, we first evaluate the radiation performance of our stealth antenna compared with the original one. Figure 5 shows the measured and simulated return loss of the stealth antenna. The associated date of original Vivaldi antenna are also presented. The measured and simulated results of the stealth antenna coincide with each other from 6 to $18 \mathrm{GHz}$ and for comparison are below $-10 \mathrm{~dB}$ over the entire X-band from $8 \mathrm{GHz}$ to $12 \mathrm{GHz}$. The return loss of the stealth antenna in X-band is only slightly larger than that of the original one, which is due to the impedance mismatch and can be further improved. In this paper, we will not focus on the feed matching design as long as the measured and simulated $S_{11}$ are acceptable. There are two resonant frequency points at nearly $9 \mathrm{GHz}$ and $12 \mathrm{GHz}$ which coincide the previous design. 


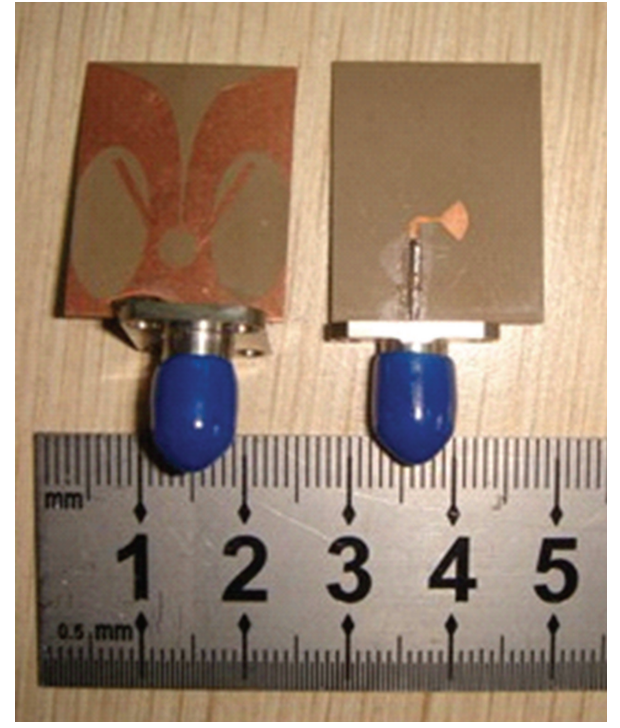

FIGURE 4: View of the fabricated stealth antenna.

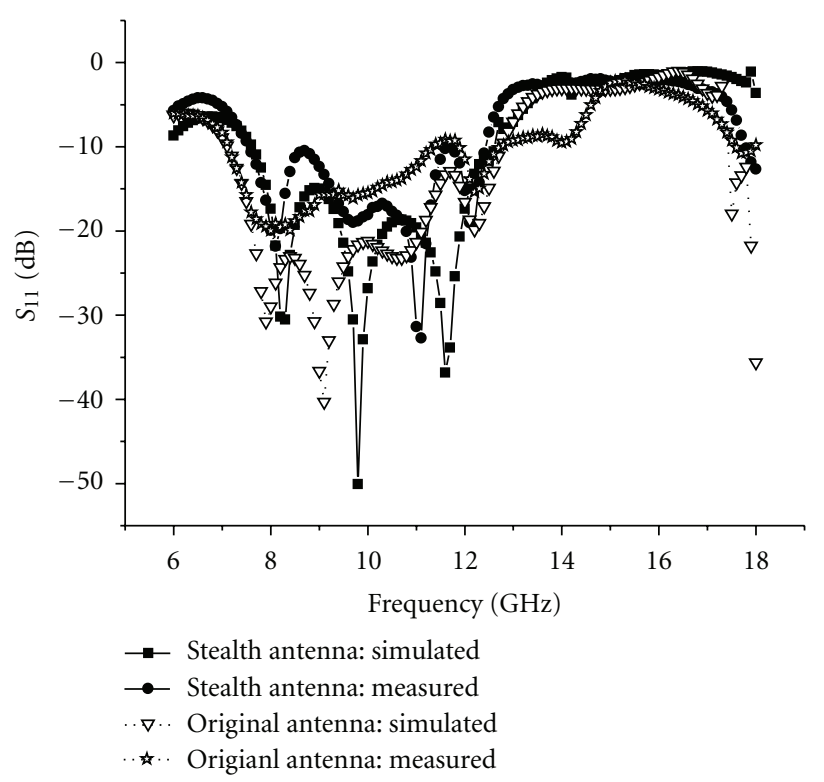

Figure 5: Return loss of the antenna.

By using the outdoor measurement method in [19], the gain and return loss of the stealth design is obtained with the transmitting and receiving antennas separated by $50 \mathrm{~cm}$. The measured and simulated antenna gains over the X-band are given in Figure 6. About $4 \mathrm{dBi}$ gain is achieved in both designs. From Figure 6 we can also observe that our stealth design method has flatter gain versus frequency as compared to the original one, which leads to a better dispersion and group delay performance.

The previous results show that our stealth design maintains good radiation performance. In the following, we will show the low observation characteristics of the stealth design. As the antenna RCSs are too low to be accurately measured in

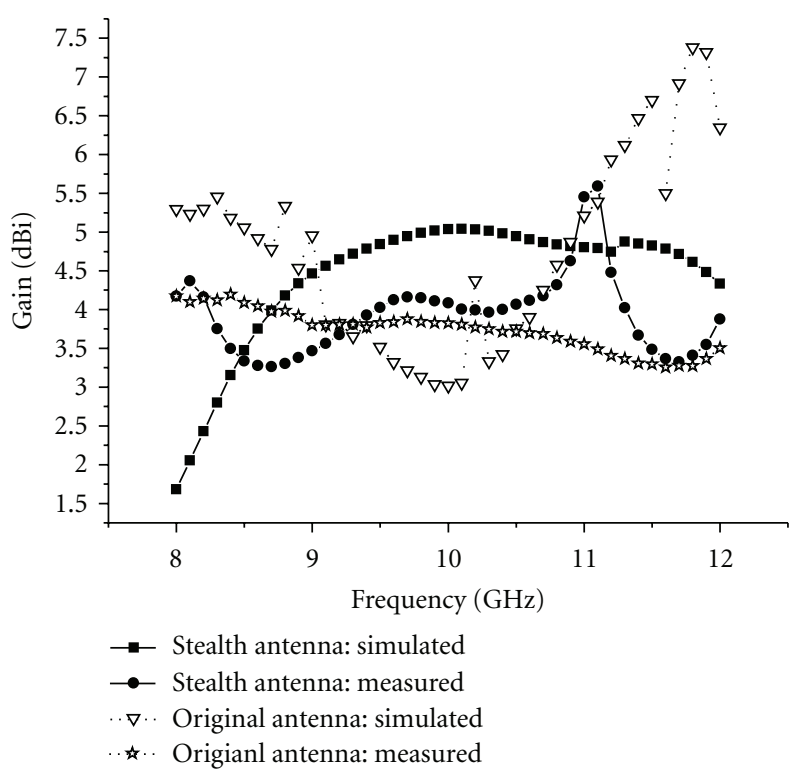

Figure 6: Gain of the antenna.

an ordinary chamber with common background noise, only simulation results are provided.

Consider a plane wave incident from the direction $\theta^{i}=0$ and $\phi^{i}=0$. As the polarization of the antenna is along the $Y$ axis, the X-polarized RCS is very small in both antenna designs as shown in Figure $7(\mathrm{a})$. However, for the copolarization in Figure 7(b), a much higher RCS is observed. As is seen in the figure, the RCS is significantly reduced by using our stealth design. Within the $\mathrm{X}$ band, the maximum RCS reduction is about $19.2 \mathrm{dBsm}$ at $11.3 \mathrm{GHz}$, and the minimum reduction is about $4.6 \mathrm{dBsm}$ at $10.1 \mathrm{GHz}$. According to the radar range equation [20], a $19.2 \mathrm{~dB}$ reduction in RCS decreases the radar detection range by $68 \%$, whereas a $4.6 \mathrm{~dB}$ reduction in RCS also can decrease the detection range by $24 \%$, which is very important in stealth design of associate platform. Meanwhile, after stealth design, the mode scattering RCS is also improved and is estimated to be about $-90 \mathrm{dBsm}$. So our stealth design method is very effective.

Figure 8 shows the bistatic RCS patterns versus scattering angle $\theta$ for $10.1 \mathrm{GHz}$ and $11.3 \mathrm{GHz}$, respectively. In both figures, the incident angle is fixed to be $\theta^{i}=0$ and $\phi^{i}=0$. It can be seen that our stealth antenna maintains much lower RCS in a wide scattering range from $0^{\circ}$ to $60^{\circ}$ and from $300^{\circ}$ to $360^{\circ}$ which are most threatening scope for stealth design. Similar RCS reduction effects are also observed at other frequencies. Compared to the scattering of dual-index Vivaldi antenna studied by Jiang fu et al. [12], our method achieves a more stable gain and wider-angle range RCS reduction.

\section{Conclusion}

In this paper, we proposed a novel low RCS design method and applied it to Vivaldi antennas. An excellent stealth performance in the entire $\mathrm{X}$-band and wide angle range 


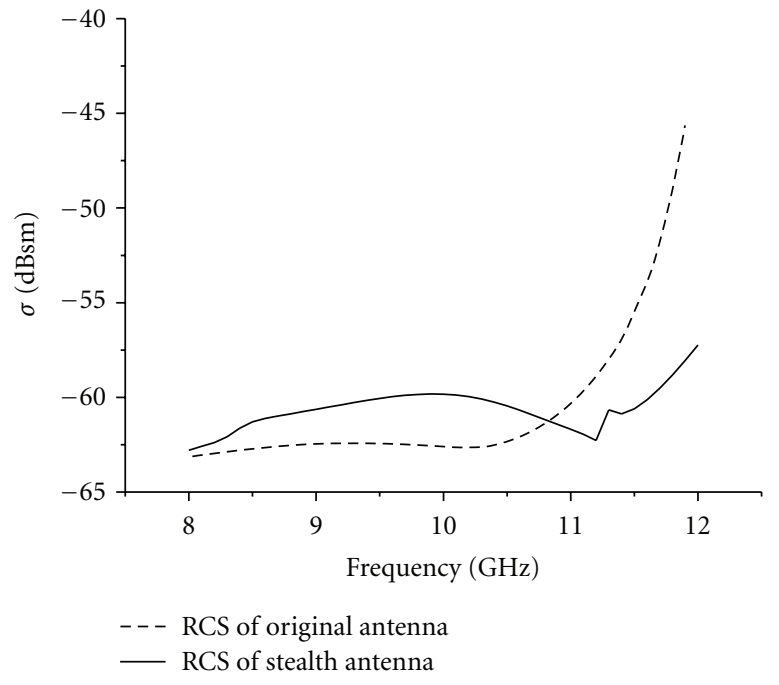

(a) X polarized

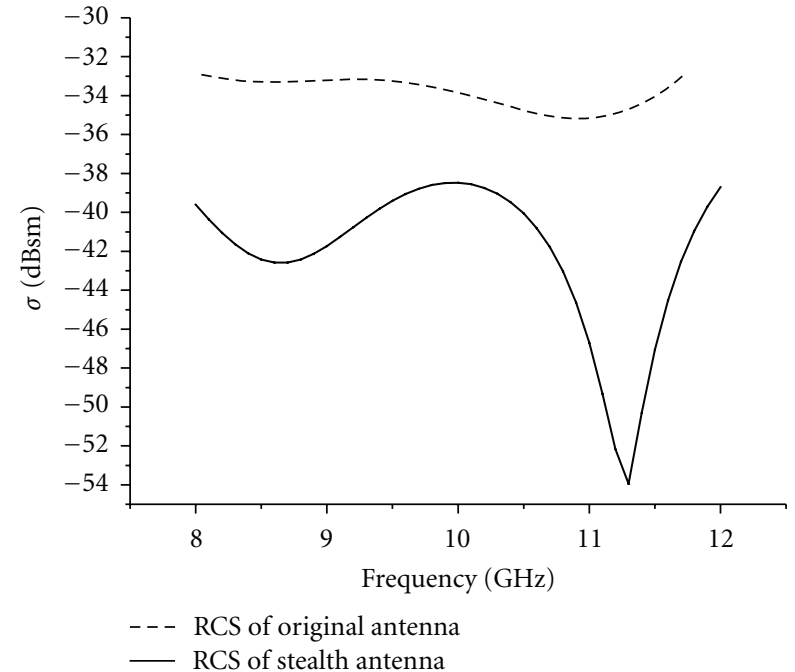

(b) Y polarized

FIgURE 7: RCS with the change of frequency.

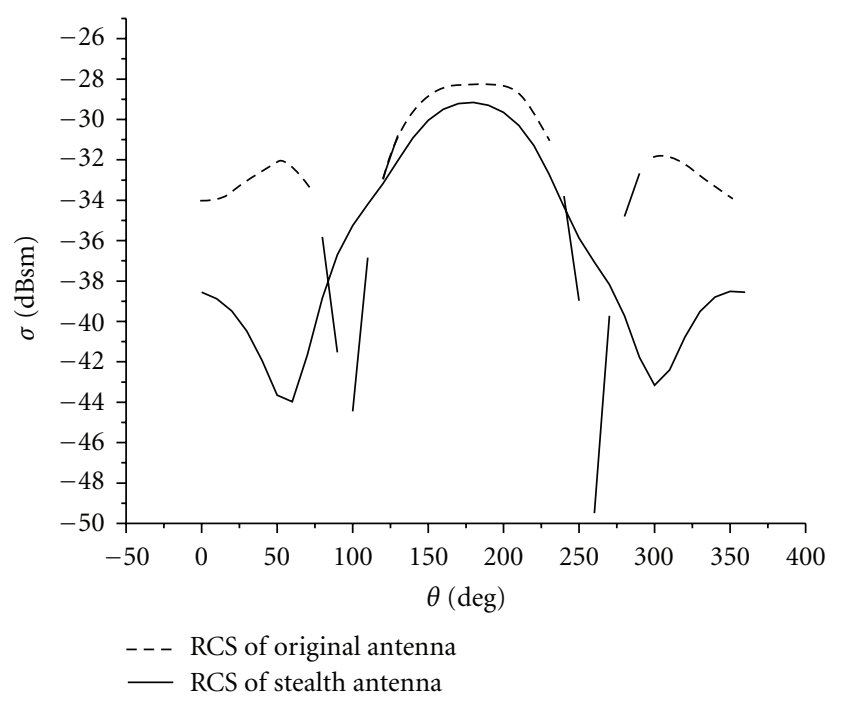

(a) At $10.1 \mathrm{GHz}$

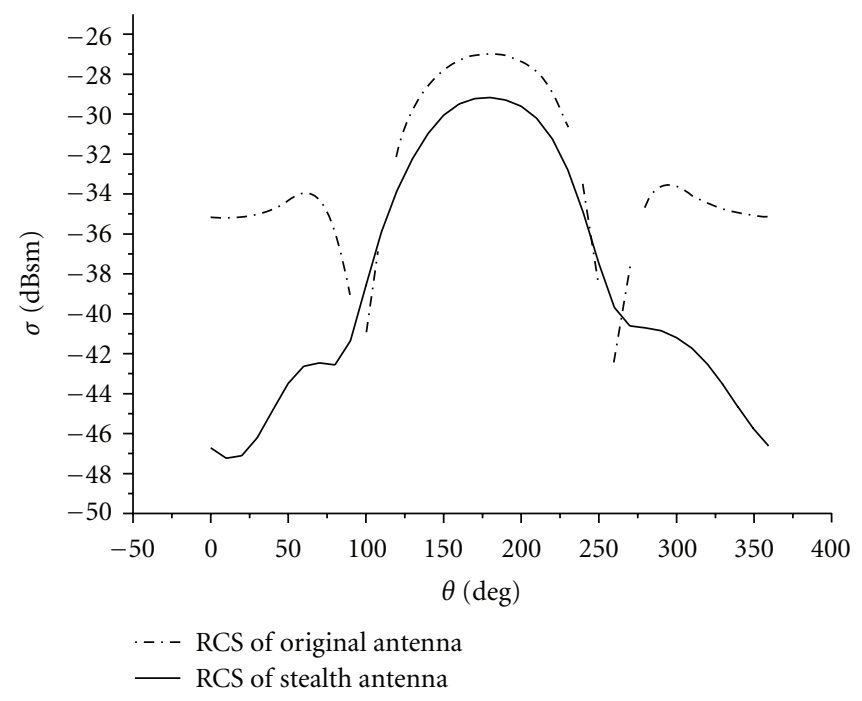

(b) At $11.3 \mathrm{GHz}$

FIGURE 8: RCS with the change of angle.

is achieved. With the proposed design method, the RCS is reduced up to $19.2 \mathrm{dBsm}$ with satisfactory radiation characteristic maintained, which shows that the proposed design is effective and potential for other microstrip antenna stealth design.

\section{Acknowledgments}

This paper is supported by the National Natural Science Foundation of China (no. 61102032), funding of State Key Laboratory of Millimeter Waves in China (Grant: K201005), the China Postdoctoral Science Foundation (no. 20110491423), the NUAA Research Funding (no.
NS2011014), and the NUAA Innovation Base Fund for Postgraduates.

\section{References}

[1] P. J. Gibson, "The Vivaldi aerial," in Proceedings of the 9th European Microwave Conference, pp. 101-105, 1979.

[2] D. H. Schaubert, S. Kasturi, A. O. Boryssenko, and W. M. Elsallal, "Vivaldi antenna arrays for wide bandwidth and electronic scanning," in Proceedings of the 2nd European Conference on Antennas and Propagation (EuCAP '07), pp. 1-6, November 2007.

[3] C. Deng and Y. J. Xie, "Design of resistive loading Vivaldi antenna," IEEE Antennas and Wireless Propagation Letters, vol. 8, pp. 240-243, 2009. 
[4] Y. Li and H. Liu, "RCS reduction of missile-borne quasitraveling wave microstrip antenna," in Proceedings of the 9th International Conference on Electronic Measurement and Instruments (ICEMI '09), pp. 3246-3249, August 2009.

[5] D. Jun, C. Chunxia, and G. ChenJiang, "A method for microstrip antenna RCS reduction," Computer Simulation, pp. 130-133, 2008.

[6] H. K. Jang, W. J. Lee, and C. G. Kim, "Design and fabrication of a microstrip patch antenna with a low radar cross section in the X-band," Smart Materials and Structures, vol. 20, no. 1, Article ID 015007, 8 pages, 2011.

[7] Y. Tan, N. Yuan, Y. Yang, and Y. Fu, "Improved RCS and efficient waveguide slot antenna," Electronics Letters, vol. 47, pp. 582-583, 2011.

[8] Y. Liu, S. X. Gong, and D. M. Fu, "A novel model for analyzing the RCS of microstrip antenna," in Proceedings of the IEEE International Antennas and Propagation Symposium and USNC/CNC/URSI North American Radio Science Meeting, pp. 835-838, June 2003.

[9] S. C. Zhao, B. Z. Wang, and Q. Q. He, "Broadband radar cross section reduction of a rectangular patch antenna," Progress in Electromagnetics Research, vol. 79, pp. 263-275, 2008.

[10] H. Y. Xu, H. Zhang, K. Lu, and X. F. Zeng, "A holly-leaf-shaped monopole antenna with low RCS for UWB application," Progress in Electromagnetics Research, vol. 117, pp. 35-50, 2011.

[11] G. Zhang, L. Xu, and A. Chen, "RCS reduction of Vivaldi antenna array using a PSS boundary," in Proceedings of the 8th International Symposium on Antennas, Propagation and EM Theory (ISAPE '08), pp. 345-347, November 2008.

[12] L. Jiang fu, G. Shu xi, Y. xue, and Z. Xiao lu, "Study of RCS on the dual-index Vivaldi antenna," Space Electronic Technology, vol. 2, pp. 26-29, 2011.

[13] L. Yang, H. Guo, X. Liu, H. Du, and G. Ji, "An antipodal Vivaldi antenna for ultra-wideband system," in Proceedings of the IEEE International Conference on Ultra-Wideband (ICUWB '10), vol. 1, pp. 301-304, September 2010.

[14] T. Li, Y. Rao, and Z. Niu, "Analysis and design of UWB Vivaldi antenna," in Proceedings of the IEEE International Symposium on Microwave, Antenna, Propagation, and EMC Technologies for Wireless Communications (MAPE'07), pp. 579-581, August 2007.

[15] M. R. Hamid, P. Gardner, P. S. Hall, and F. Ghanem, "Multimode Vivaldi antenna," Electronics Letters, vol. 46, no. 21, pp. 1424-1425, 2010.

[16] S. Gong and Y. Liu, Prediction and Reduction of Antenna Radar Cross Section, Xi' an University of Electronic Science and Technology Press, 2010.

[17] Y. T. Lo and S. W. Lee, Antenna Handbook: Theory, Applications and Design, Artech House, New York, NY, USA, 1993.

[18] B. A. Munk, Finite Antenna Arrays and FSS, Wiley-IEEE Press, 2003.

[19] G. Felic, "Antenna gain measurements in the V-band: a singleantenna method," in Proceedings of the Electromagnetic Compatibility Symposium Adelaide (EMCSA '09), pp. 98-101, September 2009.

[20] D. Lynch Jr., Introduction to RF Stealth, SciTech Publishing, 2004. 

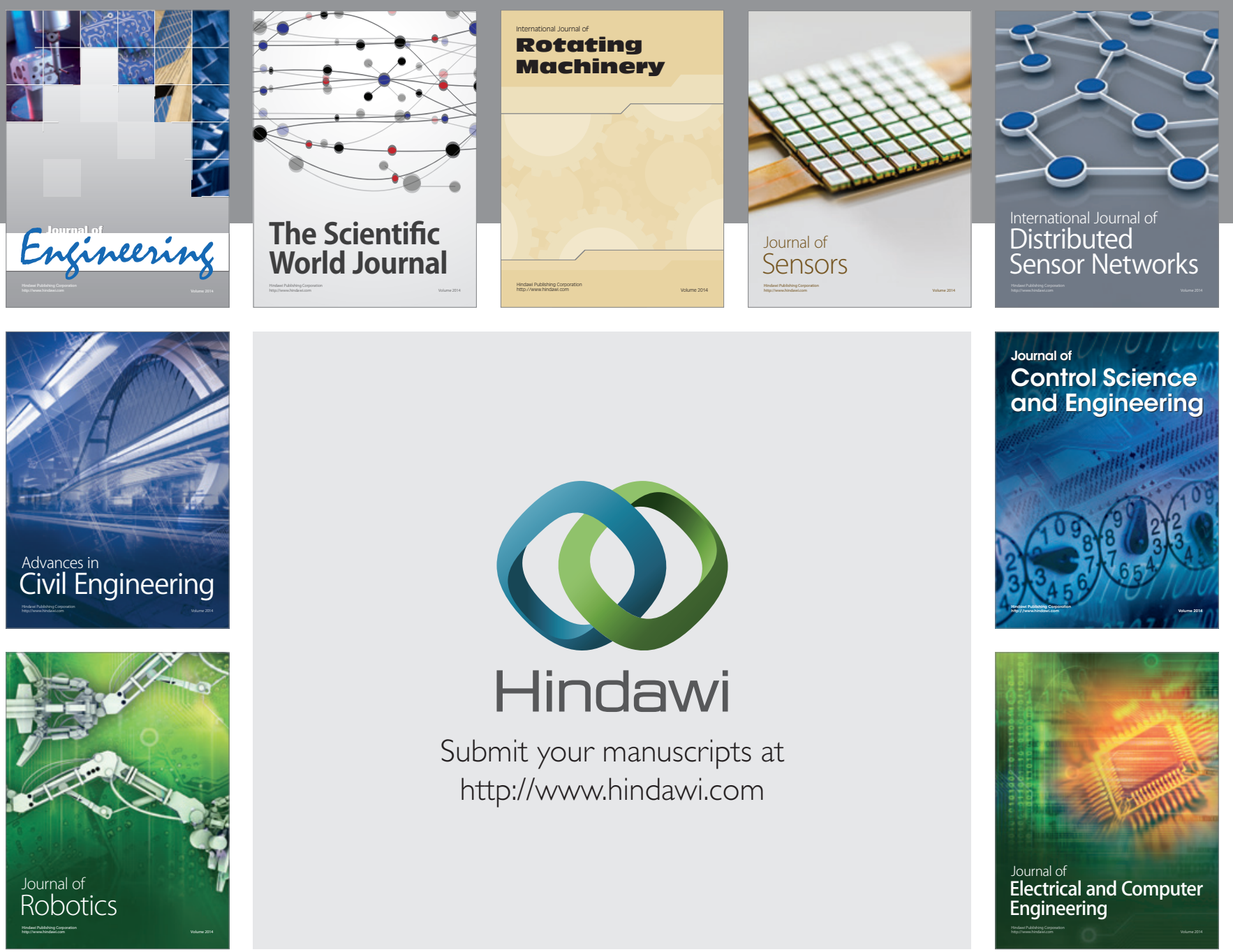

Submit your manuscripts at

http://www.hindawi.com
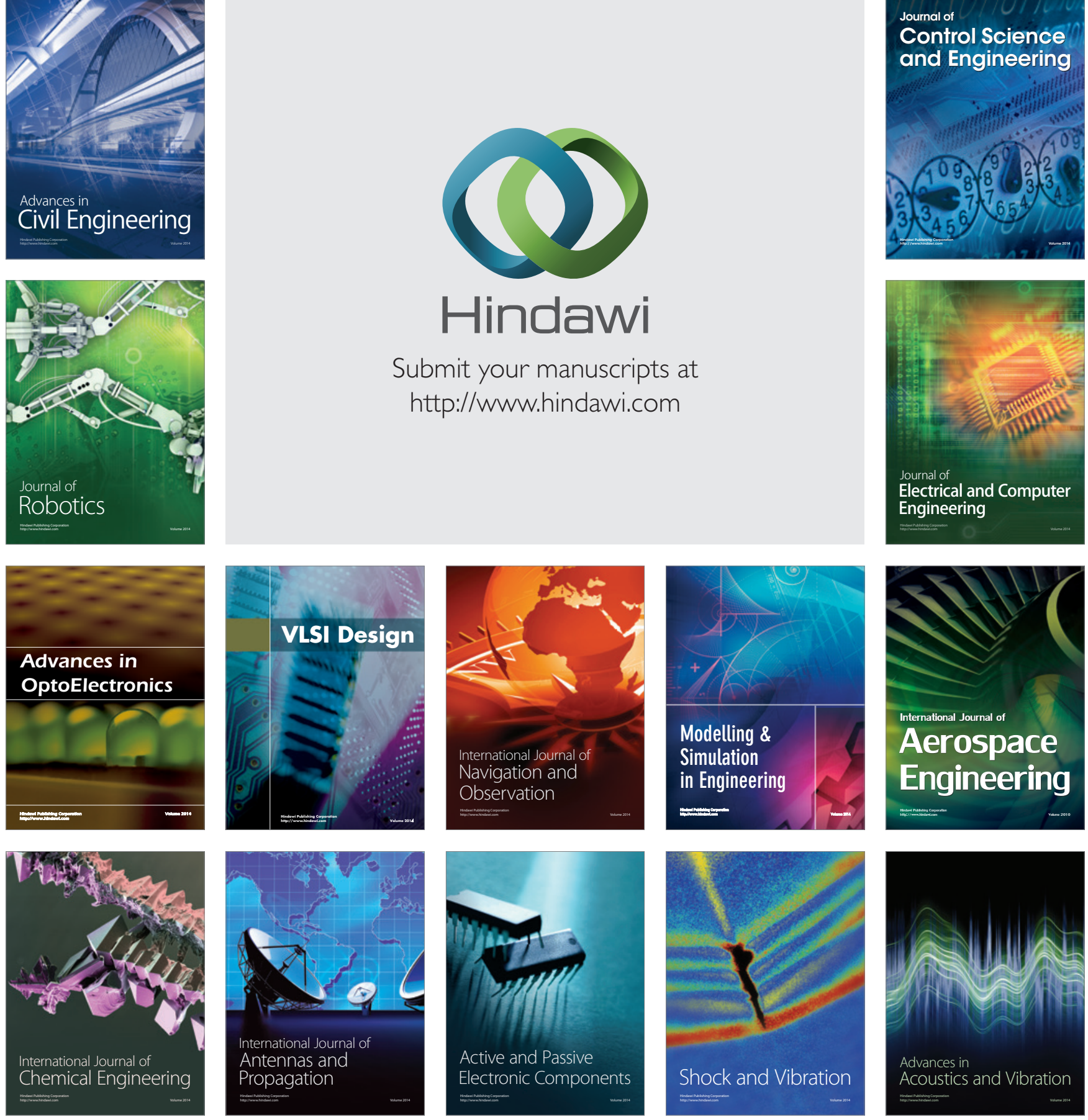